\title{
Nouvelle approche de l'évaluation de la dosimétrie extrémités à MELOX
}

\author{
H. BOREL ${ }^{1}$, M. ESPAGNAN ${ }^{2}$, B. QUESNE ${ }^{3}$, J. JEHANNO ${ }^{1}$, M. PICHEGUT ${ }^{1}$, \\ G. MARCELLIN ${ }^{2}$, F. GAUTHIER ${ }^{2}$
}

(Manuscrit reçu le 17 février 2009, accepté le 12 mai 2009)

RÉSUMÉ Les dosimétries peau et extrémités ont été mises en place afin de prévenir les effets déterministes que l'on pourrait observer suite à une exposition partielle importante. Pour être sûr de prévenir ces effets déterministes, il faut mesurer les équivalents de dose peau et extrémités au niveau des endroits les plus exposés. Pour cela, il faut d'une part connaître qu'elle est la zone la plus exposée, et, d'autre part, y placer un dosimètre approprié. C'est d'autant plus difficile lorsque le terme source est complexe et que les conditions d'expositions varient. C'est le cas pour la dosimétrie des extrémités sur l'installation de MELOX. Les différentes conditions de travail sur l'établissement de MELOX font que la zone la plus exposée des extrémités n'est pas la même pour tous les intervenants et peut varier suivant le poste de travail et le temps d'intervention. Il est donc délicat de positionner un dosimètre à l'endroit le plus exposé sachant que celui-ci varie. L'approche présentée ici consiste à caractériser l'exposition reçue par les extrémités, c'est-à-dire, « cartographier » les équivalents de dose au niveau de l'avant-bras et de la main en fonction des conditions d'exposition. À partir de ces résultats, il devient possible d'estimer l'équivalent de dose maximal perçu au niveau des extrémités à partir d'une mesure faite à un endroit précis.

ABSTRACT New approach for evaluation of dosimetry to extremities at MELOX facility.

Skin and extremity dosimetry has been set up in order to avoid deterministic effects which might be observed following an important partial exposure. For all that, dose equivalent to skin and extremities have to be measured at the most exposed areas. For that purpose, both the most expose area has to be known and an appropriate dosimeter has to be placed there. It is all the more difficult because the source term is complex and the exposure conditions can vary. It is the case for the extremity dosimetry at MELOX facility. The variety of the working conditions leads to the fact that the most exposed extremity area is not the same for all the operators and can vary due to both workstation features and duration of intervention procedures. Due to these facts, to put a dosimeter at the right position is a matter of concern. The approach presented here consists in characterizing the exposure received by extremities, i.e. in "mapping" the dose equivalents at the level of forearm and hand according to exposure conditions. Following the results obtained, the estimation of the maximum dose equivalent to extremities stemming from a measurement made at a precise point, becomes possible.

Keywords: Dosimetry / MELOX

${ }^{1}$ AREVA NC MELOX, Service Radioprotection, BP 93124, 30203 Bagnols-sur-Cèze Cedex, France.

${ }^{2}$ Laboratoire de dosimétrie, AREVA NC Marcoule, BP 761730206 Bagnols-sur-Cèze Cedex, France.

${ }^{3}$ AREVA NC, Médecin du travail, BP 93124, 30203 Bagnols-sur-Cèze Cedex, France. 


\section{Introduction}

La réglementation française en matière de radioprotection, basée sur les recommandations de la Commission Internationale de Protection Radiologique (ICRP, 1991), a imposé des limites de doses équivalentes pour certaines parties du corps. Concernant l'exposition des mains, des avant-bras, des pieds et des chevilles, la limite est fixée à $500 \mathrm{mSv}$ sur douze mois consécutifs. Il est communément appelé «extrémités » l'ensemble avant-bras, main et pieds, chevilles.

Les dosimétries Peau et Extrémités ont été mises en place afin de prévenir les effets déterministes que l'on pourrait observer suite à une exposition partielle importante. Pour être sûr de prévenir ces effets déterministes, les équivalents de dose Peau et Extrémités doivent être mesurés au niveau des endroits les plus exposés. Pour cela, il faut d'une part connaître qu'elle est la zone la plus exposée, et, d'autre part, y placer un dosimètre approprié.

Les différentes conditions de travail sur l'établissement de MELOX font que la zone la plus exposée des extrémités n'est pas la même pour tous les intervenants et peut varier suivant le poste de travail et le temps d'intervention. Il est donc délicat de positionner un dosimètre à l'endroit le plus exposé sachant que celui-ci varie. Une solution serait d'équiper chaque intervenant avec plusieurs dosimètres le long de l'avant-bras et de la main. Cependant, cette solution entrainerait une gêne certaine pour l'individu et faciliterait les ruptures de confinement (percement de gants). Pour ces raisons, cette solution n'est pas envisageable.

Une autre alternative a été étudiée. Elle consiste à caractériser l'exposition reçue par les extrémités, c'est-à-dire, « cartographier » les équivalents de dose au niveau de l'avant-bras et de la main en fonction des conditions d'exposition. L'objectif de cette étude est de définir l'équivalent de dose maximal perçu au niveau des extrémités à partir d'une mesure faite à un endroit précis.

À MELOX, les extrémités sont exposées lors du travail en boîte à gants (BàG). Il est demandé aux travailleurs, de porter un dosimètre « extrémités » au niveau du poignet, côté extérieur (côté montre). Le travail présenté ici montre comment à partir de la mesure de l'équivalent de dose au poignet, il est possible de déterminer l'équivalent de dose maximal reçu au niveau des extrémités.

Il faut noter que l'objet de ce travail n'est pas de faire une étude comparative des différents systèmes de dosimétrie, mais, à partir du système dosimétrique en place, d'évaluer au plus juste la dosimétrie extrémités. 


\section{Matériels et méthodes}

À MELOX, le personnel est confronté à un terme source qui est principalement du MOX : mélanges d'oxyde de plutonium et d'oxyde d'uranium. L'exposition due à ce terme source est principalement induite par des rayonnements photons et neutrons. L'énergie des photons varie principalement entre $13 \mathrm{keV}$ et $60 \mathrm{keV}$ et celle des neutrons, entre $0,8 \mathrm{MeV}$ et $4 \mathrm{MeV}$.

\subsection{Dosimètres utilisés}

Le dosimètre utilisé pour la surveillance des extrémités est le dosimètre extREM ${ }^{\circledR}$ fourni par le laboratoire de dosimétrie d'AREVA NC Marcoule. Il s'agit d'un dosimètre à thermoluminescence constitué de deux pastilles $\mathrm{FLi}: 1 \mathrm{~F}^{6} \mathrm{Li}$ et $1 \mathrm{~F}^{7} \mathrm{Li}$.

L'équivalent de dose photon est déterminé à partir du signal recueilli par la pastille $\mathrm{F}^{7} \mathrm{Li}$. Cette pastille est sensible aux photons d'énergie supérieure à $15 \mathrm{keV}$ et aux béta. Compte tenu des différents écrans (paires de gants) qu'il y a entre le terme source et le dosimètre et de l'énergie moyenne des béta $(\sim 100 \mathrm{keV})$, le signal induit par les béta est négligeable.

L'équivalent de dose neutron est déterminé à partir de la différence de signal entre les pastilles $\mathrm{F}^{6} \mathrm{Li}$ et $\mathrm{F}^{7} \mathrm{Li}$. La pastille $\mathrm{F}^{6} \mathrm{Li}$ est sensible aux photons, aux béta et aux neutrons. Le principe de détection des neutrons de ce dosimètre est la technique albédo. L'étalonnage de ce dosimètre vis-à-vis des neutrons a été effectué, conformément aux recommandations du rapport 66 de l'ICRU (2001), devant un assemblage MOX qui a un spectre similaire à celui présent à l'intérieur d'une boîte à gants. Une étude faite par calcul MCNP montre que le spectre d'un assemblage MOX est proche de celui d'une boîte de $\mathrm{Pu}$ « nue » qui représente le poste de travail le plus pénalisant au niveau de l'exposition des extrémités. Il y a un écart de $7 \%$ sur le coefficient de conversion moyenné sur le spectre entre un spectre «assemblage» et un spectre « $\mathrm{Pu}$ pur». Pour des raisons de confidentialité, ces spectres ne sont pas présentés.

Les conséquences de cet étalonnage sont que dans un même flux, l'équivalent de dose sur fantôme rondin est égal à l'équivalent de dose sur fantôme colonne, lui-même égal à l'équivalent de dose sur fantôme plaque. Autrement dit, face à une exposition neutron à fluence égale, les équivalents de dose doigt, poignet et organisme entier sont identiques.

Dans le rapport 66 de l'ICRU (2001), il est écrit « l'ICRU considère $H p(0,07)$ comme étant la grandeur opérationnelle également appropriée pour les neutrons ».

Des bagues $\mathrm{F}^{7} \mathrm{Li}$ on été utilisées pour effectuer des mesures d'équivalent de dose photon au niveau des doigts. Cette bague a les mêmes propriétés que la 


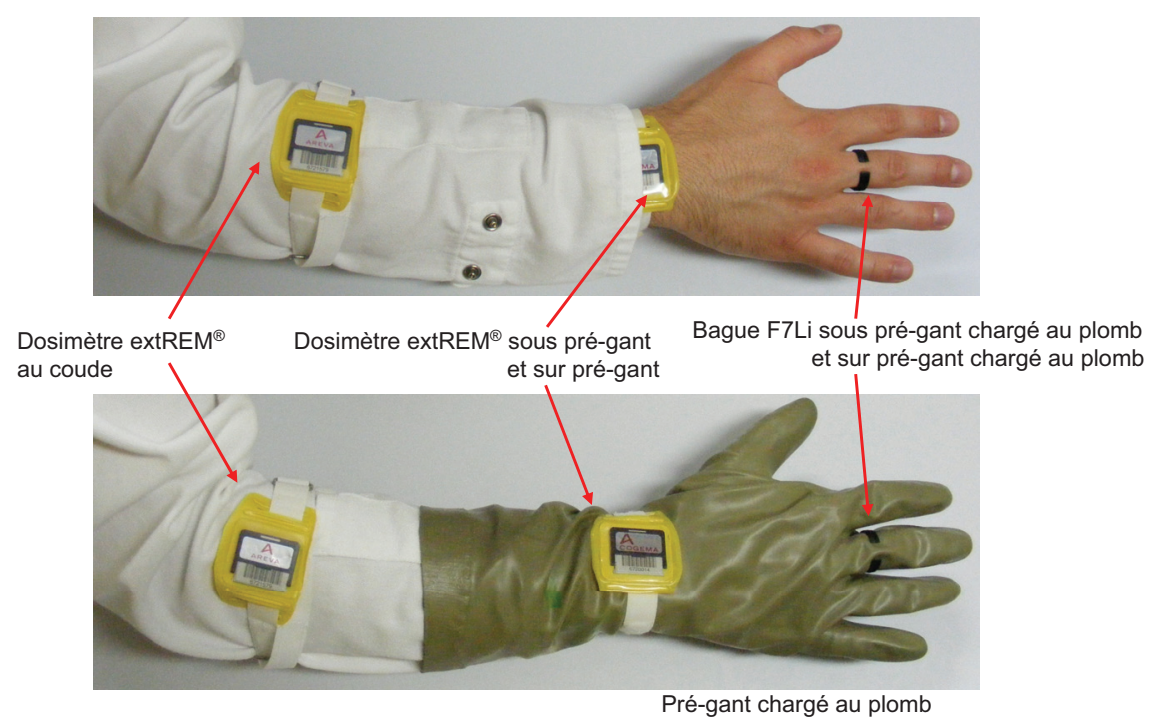

Figure 1 - Illustration du dispositif expérimental photon.

Experimental set-up for photon study.

pastille $\mathrm{F}^{7} \mathrm{Li}$ du dosimètre extREM ${ }^{\circledR}$. Le dosimètre bague, équipé du détecteur $\mathrm{F}^{7} \mathrm{Li}$ a été étalonné en fonction de l'énergie des photons sur fantôme ISO rondin par l'IRSN/SDE selon les spécifications de la norme ISO 4037-3 (2000).

Le dosimètre extREM ${ }^{\circledR}$ a été étalonné en fonction de l'énergie des photons sur fantôme ISO colonne par l'IRSN/SDE selon les spécifications de la norme ISO 4037-3 (2000).

\subsection{Méthode de caractérisation de l'exposition des extrémités aux photons}

Des individus provenant de l'ensemble de l'établissement sont équipés de 5 dosimètres ( 2 bagues $\mathrm{F}^{7} \mathrm{Li}$ et 3 extREM ${ }^{\circledR}$ ), voir figure 1.

Suivant les interventions à réaliser, le port de pré-gants chargés au plomb est rendu nécessaire. Ils offrent une protection radiologique en atténuant d'un facteur 5 les photons. Mais leurs ports entraînent une perte importante de dextérité qui fait qu'ils ne sont pas portés systématiquement partout et par tout le monde. Il faut noter que ces pré-gants ne protègent pas l'ensemble des extrémités, ils ne montent pas plus haut que la mi-avant-bras. 


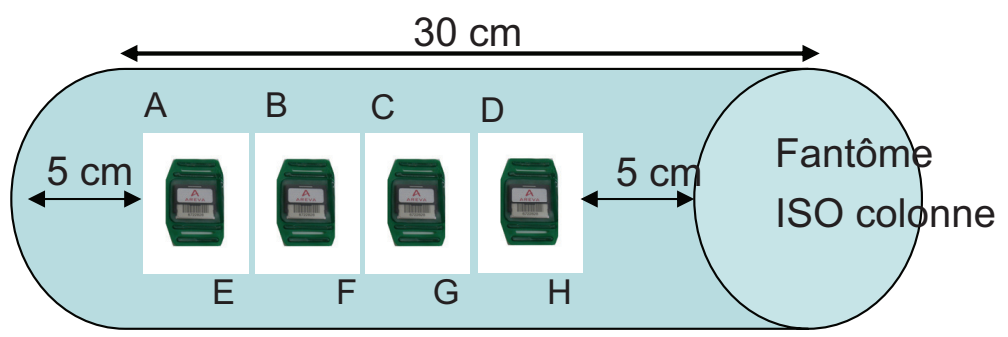

Figure 2 - Illustration du dispositif expérimental neutron.

Experimental set-up for neutron study.

Ces 5 dosimètres constituent un lot. Le port de 5 dosimètres étant contraignant pour les personnes, un individu est équipé d'un lot de dosimètre le temps d'une intervention. Puis ce même lot de dosimètres est porté par un autre individu intervenant sur le même poste de travail. Et ainsi de suite jusqu'à ce que l'équivalent de dose minimal estimé sur un dosimètre du lot soit supérieur à $0,5 \mathrm{mSv}$.

Un lot de dosimètres constitue une mesure. Cinq mesures sont effectuées par secteur de travail. MELOX comporte deux gros secteurs de travail: la zone «poudres» et la zone «pastilles». Chacune de ces zones est constituée par plusieurs boîte à gants.

Chaque dosimètre a été clairement identifié pour être positionné toujours au même endroit. À chaque lot était associée une feuille d'expérimentation où étaient reporté, le lieu précis de chaque intervention, le débit de dose au niveau du rond de gant et le temps d'intervention.

Au final, une quarantaine de personnes ont participé à ces mesures de dosimétrie extrémités et ces tests ont eut lieu sur une douzaine de postes de travail.

\subsection{Méthode de caractérisation de l'exposition des extrémités aux neutrons}

Sur un fantôme colonne [ISO 4037-3], 8 dosimètres ont été disposés : 4 d'un côté et 4 diamétralement opposés (Fig. 2). Les dosimètres sont placés à minima à $5 \mathrm{~cm}$ des bords pour éviter les effets de bords.

Le fantôme ainsi équipé est ensuite recouvert par une feuille de plomb afin de diminuer le signal induit par les photons.

Ce dispositif est ensuite placé dans un gant de boîte à gants. Le fantôme est positionné au niveau d'un poste de travail en formant un angle de $45^{\circ}$ environ avec le panneau de la boîte à gants. 


\section{TABLEAU I}

Équivalent de dose photon $H p(0,07)$ au doigt et au poignet protégés par un pré-gant chargé au plomb.

Dose équivalent $H p(0.07)$ measured at finger and wrist protected by leaded-glove and at the elbow for photon irradiation.

\begin{tabular}{lccccc}
\hline & \multicolumn{5}{c}{ mesures sous le pré-gant en plomb } \\
\cline { 2 - 6 } & $\begin{array}{c}\text { dose au doigt } \\
{[\mathrm{mSv}]}\end{array}$ & $\begin{array}{c}\text { dose au poignet } \\
{[\mathrm{mSv}]}\end{array}$ & $\begin{array}{c}\text { dose au coude } \\
{[\mathrm{mSv}]}\end{array}$ & ratio doigt/poignet & ratio coude/poignet \\
\hline secteur poudre & 0,81 & 0,66 & 1,39 & 1,22 & 2,10 \\
& 0,65 & 0,41 & 0,54 & 1,59 & 1,32 \\
& 0,88 & 0,83 & 2,98 & 1,05 & 3,57 \\
\hline secteur pastille & 0,51 & 0,48 & 1,62 & 1,06 & 3,38 \\
& 0,90 & 0,68 & 2,64 & 1,32 & 3,88 \\
\hline & 0,57 & 0,42 & 1,14 & 1,36 & 2,71 \\
& 0,22 & 0,17 & 0,61 & 1,29 & 3,59 \\
& 1,03 & 0,91 & 2,80 & 1,13 & 3,08 \\
& 0,71 & 0,87 & 2,44 & 0,82 & 2,80 \\
\hline & 0,64 & 0,46 & 1,19 & 1,39 & 2,59 \\
\hline & & moyenne & 1,22 & 2,90 \\
\hline
\end{tabular}

Ce test est effectué sur plusieurs postes de travail. Avec ce dispositif, nous pouvons étudier le signal neutron suivant l'axe du cylindre (fantôme colonne) représentant l'avant-bras d'un opérateur.

\section{Résultats}

\subsection{Résultats de la caractérisation de l'exposition des extrémités aux photons}

Ces tests ont permis d'étudier la répartition des équivalents de dose le long de l'avant-bras et de la main, sur l'ensemble de l'installation où la dosimétrie extrémités est significative, et en fonction des conditions d'exposition : port ou non de pré-gant chargés au plomb.

Les résultats de l'exposition des extrémités en présence de pré-gants chargés au plomb sont présentés dans le tableau I.

Les résultats de l'exposition des extrémités en absence de pré-gants chargés au plomb sont présentés dans le tableau II. 
TABLEAU II

Équivalent de dose photon $H p(0,07)$ au doigt et au poignet non protégés par un pré-gant chargé au plomb.

Dose equivalent $H p(0.07)$ values measured at finger, wrist and elbow without leaded-glove protection for photon irradiation.

\begin{tabular}{cccccc}
\hline & \multicolumn{5}{c}{ mesures sur le pré-gant en plomb } \\
\cline { 2 - 6 } & $\begin{array}{c}\text { dose au doigt } \\
{[\mathrm{mSv}]}\end{array}$ & $\begin{array}{c}\text { dose au poignet } \\
{[\mathrm{mSv}]}\end{array}$ & $\begin{array}{c}\text { dose au coude } \\
{[\mathrm{mSv}]}\end{array}$ & ratio doigt/poignet & ratio coude/poignet \\
\hline secteur poudre & 3,90 & 3,78 & 1,39 & 1,03 & 0,37 \\
& 5,00 & 2,00 & 0,54 & 2,50 & 0,27 \\
& 4,93 & 2,95 & 2,98 & 1,67 & 1,01 \\
\hline secteur pastille & 2,31 & 2,25 & 1,62 & 1,03 & 0,72 \\
& 4,54 & 3,65 & 2,64 & 1,24 & 0,72 \\
\hline & 2,00 & 2,09 & 1,14 & 0,96 & 0,55 \\
& 1,22 & 0,61 & 0,61 & 2,00 & 1,00 \\
& partie en déchêts & 3,83 & 2,80 & - & 0,73 \\
& 4,21 & 4,00 & 2,44 & 1,05 & 0,61 \\
\hline & 2,98 & 2,71 & 1,19 & 1,10 & 0,44 \\
\hline & & incertitude relative à $2 \sigma$ & 1,40 & 0,64 \\
\hline
\end{tabular}

Ces résultats montrent qu'il n'y a pas de différence significative entre les valeurs du secteur poudres et celles du secteur pastilles.

Le fait de porter des pré-gants chargés au plomb entraîne une exposition maximale au niveau de la zone non protégée : le coude (Tab. I). L'équivalent de dose au coude est de l'ordre de 3 fois supérieur à celui mesuré au poignet. Les rapports équivalent de dose doigt sur équivalent de dose poignet sont généralement supérieur à 1 (1,22 en moyenne). La région palmaire est donc plus exposée que le poignet.

En absence de pré-gants (Tab. II), l'exposition maximale est observée au niveau de la région palmaire. Le rapport des équivalents de dose doigt sur poignet est égal à 1,40 en moyenne. Mais sur certain poste de travail, ce rapport peut monter à 2,00-2,50. En revanche, la région du coude est dans cette configuration, moins exposée que le poignet.

À partir de cette caractérisation de l'exposition des extrémités, il est possible de déterminer l'équivalent de dose maximal reçu au niveau de l'avant-bras et de la main. De manière conservative, il sera finalement appliqué un facteur 3 sur l'équivalent de dose mesuré au poignet pour donner l'équivalent de dose extrémités. 


\section{TABLEAU III}

Signal neutron moyen mesuré le long de la génératrice du fantôme colonne. Average neutron signal measured along the pillar phantom.

\begin{tabular}{lccc}
\hline & poste 1 & poste 2 & poste 3 \\
\hline $\mathrm{x}$ & signal moyen $(\mathrm{gu})$ & signal moyen $(\mathrm{gu})$ & signal moyen $(\mathrm{gu})$ \\
\hline $\mathrm{A}+\mathrm{E}$ & 51,74 & 121,34 & 49,27 \\
$\mathrm{~B}+\mathrm{F}$ & 38,31 & 139,58 & 46,71 \\
$\mathrm{C}+\mathrm{G}$ & 35,46 & 154,33 & 62,35 \\
$\mathrm{D}+\mathrm{H}$ & 48,84 & 161,79 & 69,88 \\
\hline moyenne & 43,59 & 144,26 & 57,05 \\
incertitude relative à $2 \sigma$ & $29 \%$ & $20 \%$ & $31 \%$ \\
\hline
\end{tabular}

Le laboratoire de dosimétrie d'AREVA NC Marcoule ayant suivit le déroulement de tous ces tests, va intégrer ce facteur 3 (FT $\gamma$ ), pour les photons, lors du traitement des dosimètres extREM provenant de MELOX.

\subsection{Résultats de la caractérisation de l'exposition des extrémités aux neutrons}

Les résultats des mesures effectuées sur 3 postes de travail sont présentés dans le tableau III. L'analyse se fait directement sur les signaux restitués par les dosimètres. L'objet de ces mesures est d'étudier l'homogénéité du signal neutron le long de l'axe du fantôme colonne.

L'analyse statistique utilisée est le test du carré des différences successives (CETAMA, 1986). L'hypothèse $\mathrm{H}_{0}$ suivante a été testée : le signal neutron ne présente pas de variation systématique entre la tête et le pied du fantôme colonne.

L'analyse statistique a finalement conclu qu'au niveau de probabilité de $99 \%$, on peut admettre l'homogénéité du signal neutron mesuré sur fantôme colonne. Autrement dit, il n'y a pas de différence significative du signal neutron suivant l'axe du cylindre.

Toutefois, il faut garder à l'esprit que la mesure d'un signal neutron à l'aide d'un dosimètre à albédo, sur une masse faible (fantôme colonne), n'est pas la meilleure technique.

Le signal neutron semble homogène sur l'ensemble du fantôme colonne, donc, le flux de neutron peut être considéré comme homogène sur cette zone. Autrement dit, l'avant-bras et la main sont exposés à une fluence de neutron homogène au niveau d'un poste de travail, à l'intérieur d'une boîte à gants. 
Le dosimètre extREM ${ }^{\circledR}$ étant étalonné en parfait accord avec les recommandations 66 de l'ICRU (2001), dans un même flux de neutrons, les équivalents de dose au doigt, au poignet et au coude sont égaux.

Les conséquences de ces résultats est que l'équivalent de dose extrémités neutron peut être considéré comme homogène en tout point de l'avant-bras et de la main.

\section{Conclusion}

Ce travail a permis de caractériser l'exposition de l'avant-bras et de la main d'un travailleur intervenant en boîte à gants sur l'installation de MELOX. À partir des résultats présentés dans le chapitre précédent, l'établissement de l'équivalent de dose extrémité se fait de la manière suivante :

$$
H p(0,07)_{\text {Extrémités }}=H p(0,07)_{\text {poignet, } \gamma} F T \gamma+H p(0,07)_{\text {poignet }, n}
$$

avec $\mathrm{FT} \gamma=3$,

$H p(0,07)_{\text {poignet }}$ neutron et photon sont déterminés à partir du dosimètre extREM ${ }^{\circledR}$ portés au poignet.

Notre méthode permet d'établir l'équivalent de dose maximal reçu au niveau des extrémités. Il ne faut pas oublier que la dosimétrie extrémités est surveillée dans le but de prévenir tout effet déterministe et, par conséquent, il est nécessaire de déterminer l'équivalent de dose maximal.

Ce travail ne peut se faire qu'à partir d'une bonne connaissance du terme source et des conditions d'exposition. Les dosimètres doivent être correctement calibrés par rapport au terme source au poste de travail. Ceci est d'autant plus vrai lors d'expositions dues aux neutrons (étalonnage par rapport au spectre neutronique de l'installation). Ce travail doit se faire en étroite collaboration entre la PCR et le laboratoire de dosimétrie afin que celui-ci puisse valider les expérimentations et intégrer les résultats (FT $\gamma$, par exemple) dans sa méthodologie d'établissement des doses. Ce travail a abouti à la signature d'un protocole entre le médecin du travail, le laboratoire de dosimétrie d'AREVA NC Marcoule et le service de Radioprotection de MELOX, sur la méthodologie d'établissement des équivalent de dose extrémités.

Ce travail illustre l'importance d'une collaboration étroite entre les équipes de radioprotection, le médecin du travail et les responsables du laboratoire de dosimétrie (Fig. 3). Chacun par sa connaissance et son expérience apporte les éléments utiles à la bonne élaboration de dose individuelle. Ce type de travail 


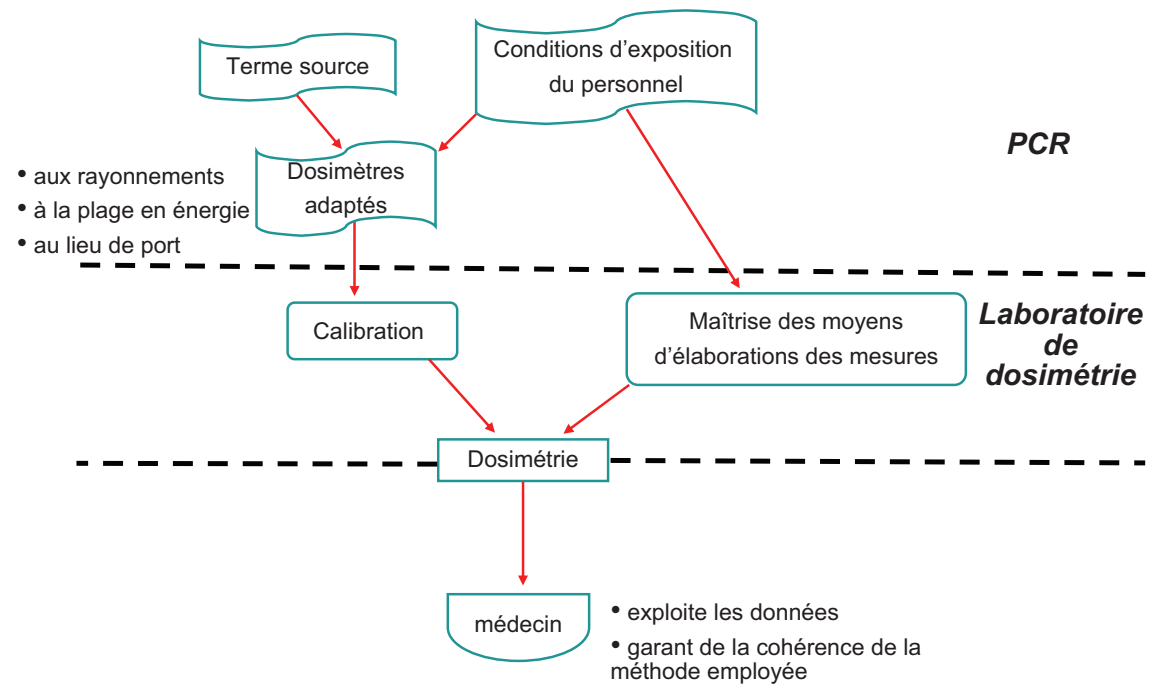

Figure 3 - Illustration de la collaboration mise en place pour une bonne élaboration de la dosimétrie. Actors of the system for a good dosimetry evaluation.

réalisé sur l'établissement MELOX peut être mené sur un autre établissement nucléaire avec des moyens adaptés, bien que chaque établissement possède des caractéristiques d'exposition qui lui sont propres. Une démarche de même nature nous paraît utile et souhaitable dans les installations qui présentent un risque d'exposition significative aux extrémités, et qui ne l'aurait pas encore entreprise.

\section{RÉFÉRENCES}

ICRP Publication 60 (1991) 1990 Recommendations of the International Commission on Radiological Protection, Ann. ICRP 21(1-3).

ICRU Publication 66 (2001) Recommendations on the International Commission on Radiation Units and Measurements, pp. 57-58.

ISO 4037-3 (2000) Rayonnement X et gamma de référence pour l'étalonnage des dosimètres et des débitmètres et pour la détermination de leur réponse en fonction de l'énergie des photons Partie 3 - AFNOR

CETAMA (1986) « Recherche d'une variable continue », dans Statistique appliquée à l'exploitation des mesures, $2^{\mathrm{e}}$ édition. Masson, Paris. 